\title{
On Relay Selection in Bursty Impulsive Noise Channel
}

\author{
Md. Sahabul Alam \\ Department of Electrical and Computer Engineering \\ McGill University, Montreal, Canada \\ Email: md.s.alam@mail.mcgill.ca
}

\author{
Fabrice Labeau \\ Department of Electrical and Computer Engineering \\ McGill University, Montreal, Canada \\ Email: fabrice.labeau@mcgill.ca
}

\begin{abstract}
Relay selection among available relays is crucial in improving the performance of cooperative relaying. In this paper, we address the relay selection for a multi-relay decode-andforward (DF) cooperative relaying scheme over channels impaired by bursty impulsive noise in the presence of Rayleigh fading. For this scheme, we propose a novel best relay selection technique which is based on both the channel state information of sourcerelay and relay-destination links and the state of the impulsive noise that affect those links. Then, we provide a end-to-end bit error rate (BER) expression for the proposed scenario using BPSK modulation. Finally, the accuracy of the provided analytical results and the effectiveness of the proposed best relay selection algorithm under different network topology is discussed. It is shown that, by using the bursty impulsive noise characteristics, the proposed relay selection algorithm performs significantly better than the conventional relay selection algorithm which is optimized for additive white Gaussian noise environment.
\end{abstract}

\section{INTRODUCTION}

Cooperative relaying (CR) has been identified as a promising technology since last decade due to its reliability over fading and interference channels [1], [2]. Relay selection among available relays is crucial in improving the performance of CR. There exists a rich body of literature on relay selection techniques in $\mathrm{CR}$, where the relay nodes are selected according to some well-defined system parameters. Blestas et al. [3] have proposed a best relay selection criterion, where it is assumed that the set of relays which are able to decode the source symbol successfully are considered for best relay selection. For this scheme, the relay nodes monitor the instantaneous channel conditions toward the source and the destination. The best relay is then selected in a distributed fashion based on the minimum or the harmonic mean of source-relay $(S R)$ and relay-destination $(R D)$ channel gains. The authors in [4] have introduced another best relay selection method based on a metric, which is the instantaneous scaled harmonic mean function of its $S R$ and $R D$ channel gains. The rationale behind this protocol is that if the direct link between the sourcedestination $(S D)$ pair have high quality, there is no need for the relay to forward the source information. Since the relay is not always transmitting, this scheme achieves a significant improvement in bandwidth efficiency. In [5], the authors have proposed another best relay selection method in which the destination node chooses the best relay based on the minimum of $S R$ and $R D$ link signal-to-noise ratios (SNRs) and allows the selected relay to cooperate only if the minimum of its $S R$ and $R D$ link SNRs is greater than the SNR of the direct link. While [4] considered that the optimal relay re-transmits in the second phase only if its correctly decoded, possible error propagation from the optimal relay is considered in [5].

However, all of the above best relay selection techniques assume that the relays and destination are impaired by additive white Gaussian noise (AWGN) only. In practice, wireless systems are impaired by non-Gaussian noise and interference also [6]. For example, due to partial discharge and switching effects impulsive noise is observed in power substations, around power transmission lines, and in some mobile radio scenarios [6]-[8]. Also, the impulsive noise measurement campaigns show that in most cases the impulses occur in bursts [7], [8]. The authors in [9] have extended the conventional optimal $\max -\min$ relay selection criterion for interference limited environment. It is shown that the presence of interference modifies the statistical description of the selection process and incurs heavy penalties when the conventional relay selection criteria are utilized. While [9] considered various best relay selection for cooperative systems impaired by Gaussian interference, the authors in [10] proposed a new best relay selection criterion based on the derived asymptotic error rate expressions in the presence of generic noise. It is shown that compared to Gaussian case, the best relay selection in non-Gaussian noise do depend on the type of noise via certain noise moments.

In this paper, we investigate the performance of relay selection techniques in bursty impulsive noise environment modeled by a two-state Markov-Gaussian (TSMG) process. The analysis of [9], [10] assumes that the interfering signals manifest during the entire duration of transmission. However, in our noise model, the impulses occur only with a probability $p_{B}$, and will not be present at all times. In general, it would be a more likely scenario that harmful impulses are not always present. We propose a novel relay selection technique which is based on both the channel state information of source-relay and relay-destination links and the state of the impulsive noise that affect those links. To know the states of the impulsive noise, we propose a state detection algorithm that can exploit the memory of the impulsive noise through maximum a posteriori (MAP) detection [11]. It is shown that the proposed MAP-based relay selection algorithm performs significantly better than the conventional relay selection algorithm which is optimized for AWGN environment, and which neglects the noise memory.

The rest of the paper is organized as follows. In Section II, the system model is introduced and Section III provides an overview of two-state Markov-Gaussian process. In Section IV, we introduce the relay selection techniques and Section $\mathrm{V}$ provides the mathematical framework for the proposed 


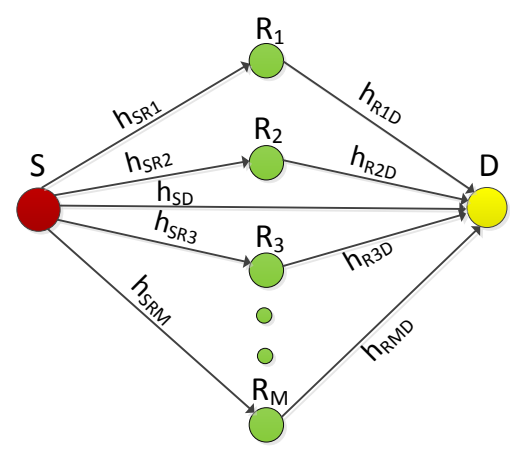

Fig. 1. Cooperative communication with multiple relays.

scenario. Section VI provides the performances in terms of BER and finally, some conclusions are drawn in Section VII.

\section{SYSTEM MODEL}

Here, we consider a DF CR scheme with $M$ relays, as shown in Fig 1, where the data transmission between the $S D$ pair is assisted by those relays. We assume that all nodes are equipped with a single antenna and share the same bandwidth for data transmission. We also assume that each node operates in half-duplex mode. The cooperative communication takes place in two time slots. In the first time slot, $S$ transmits the data to $D$, and due to the broadcast nature of the wireless channel, the relays also receive it. The selected relay then decodes the received signal and retransmits its decoded information to the destination in the second time slot.

Consider that the source $S$ generates a frame of binary information of length $K$ bits $\left(b_{0}, b_{1}, \ldots, b_{K-1}\right)$, mapped into a BPSK modulated sequence $\left(x_{S, 0}, x_{S, 1}, \ldots, x_{S, K-1}\right)$, and transmitted to both the relays and the destination in the first time slot. The signals received at relay $R_{m}, R_{m} \in$ $\left\{R_{1}, R_{2}, \ldots, R_{M}\right\}$ and $D$ at each time epoch $k, k=$ $0,1, \ldots, K-1$ can be respectively expressed as

$$
\begin{aligned}
y_{S R_{m}, k} & =\sqrt{\mathrm{P}_{S}} h_{S R_{m}, k} x_{S, k}+n_{S R_{m}, k} \\
y_{S D, k} & =\sqrt{\mathrm{P}_{S}} h_{S D, k} x_{S, k}+n_{S D, k}
\end{aligned}
$$

where $\mathrm{P}_{S}$ is the average source transmission power for each symbol, $x_{S, k}$ is the transmitted symbol from $S, h_{i j, k}$ is the channel coefficient for the $i j$ link, $i \in\left(S, R_{m}\right)$ and $j \in\left(R_{m}, D\right)$, and $n_{i j, k}$ is the noise term for the $i j$ link that captures the combined effects of AWGN and the impulsive interferers. We refer to the scenario where the destination is affected by AWGN only and the relays are affected by impulsive interferers also. We assume independent Rayleigh fading in all links, i.e., for each $i j$ link, $h_{i j}$ is modeled as a zero-mean, independent, circularly symmetric complex Gaussian random variable with variance $\Omega_{i j} \equiv \varepsilon\left\{\left|h_{i j}\right|^{2}\right\}=1 / \lambda_{i j}^{\eta}$, where $\varepsilon\{\cdot\}$ denotes expectation operator, $\lambda_{i j}$ is the relative distance of $i$ from $j$, and $\eta$ is the path loss exponent. The noise sample $n_{i j, k}$ is modeled as a TSMG process, which is in fact a Markov process in which the marginal distribution in each state are Gaussian. In the following section we will provide an overview of the model and explain the physical significance of each parameter.
In the second time slot, the received signal $y_{S R_{b}}$ at the best relay $R_{b}$ is passed through a demodulator to recover the source information. In section IV, we describe how the best relay is selected among all the available relays. The best relay then decodes the source information, potentially making an error. After recovering the source information, the relay modulates it using the same modulation format as in $S$ and forwards it to the destination with average transmission power $\mathrm{P}_{b}$. The signal received at the destination in this case is given by

$$
y_{R_{b} D, k}=\sqrt{\mathrm{P}_{b}} h_{R_{b} D, k} x_{R_{b}, k}+n_{R_{b} D, k}
$$

where $x_{R_{b}, k}$ is the transmitted signal from $R_{b}$ which may or may not be equal to $x_{S, k}$.

\section{AN OVERVIEW OF TSMG MODEL}

A TSMG model is introduced by Fertonani [12] to characterize the bursty impulsive noise. At each time epoch $k$, the statistical properties of the noise sample $n_{i j, k}$ are completely defined by the channel state $s_{k}, s_{k} \in\{G, B\}$. In our noise modeling, $G$ stands for the good channel that is impaired by the background Gaussian noise only and $B$ for the bad channel which is impaired by impulsive interferers also. For each $i j$ link, we model $n_{i j, k}$ as a zero-mean, independent, circularly symmetric complex Gaussian random variable with variances depending on $s_{k}$, so that conditioned on $s_{k}$, the probability density functions (PDFs) of $n_{i j, k}$ can be expressed as

$$
\begin{aligned}
p\left(n_{i j, k} \mid s_{k}=G\right) & =\frac{1}{\pi \sigma_{G}^{2}} \exp \left(-\frac{\left|y_{i j, k}-\sqrt{\mathrm{P}_{i}} h_{i j, k} x_{i, k}\right|^{2}}{\sigma_{G}^{2}}\right)(4) \\
p\left(n_{i j, k} \mid s_{k}=B\right) & =\frac{1}{\pi \sigma_{B}^{2}} \exp \left(-\frac{\left|y_{i j, k}-\sqrt{\mathrm{P}_{i}} h_{i j, k} x_{i, k}\right|^{2}}{\sigma_{B}^{2}}\right)(5)
\end{aligned}
$$

where $\sigma_{G}^{2}$ and $\sigma_{B}^{2}$ are the average noise power of the good channel and bad channel respectively. The statistical description of the state process $s^{K}=\left\{s_{0}, s_{1}, \ldots, s_{K-1}\right\}$ completely characterizes the channel and, for Markov-Gaussian model, $s^{K}$ is expressed as a stationary first-order Markov-process with

$$
p\left(s^{K}\right)=p\left(s_{0}\right) \prod_{k=0}^{K-1} p\left(s_{k+1} \mid s_{k}\right)
$$

for each realization of the process. Therefore, the state process is described by the state transition probabilities $p_{s_{k} s_{k+1}}=$ $p\left(s_{k+1} \mid s_{k}\right), s_{k}, s_{k+1} \in\{G, B\}$. From the state transition probabilities, the stationary probabilities $p_{G}$ and $p_{B}$ of being in $G$ and $B$ state are respectively given by [12],

$$
p_{G}=\frac{p_{B G}}{p_{G B}+p_{B G}}, \quad \text { and } \quad p_{B}=\frac{p_{G B}}{p_{G B}+p_{B G}}
$$

where $p_{B G}$ denotes the transition probability from state $B$ to state $G$ and similarly $p_{G B}$ is the transition probability from $G$ to $B$. Also, the parameter $\mu=\frac{1}{p_{G B}+p_{B G}}$ quantifies the noise memory, with $\mu=1$ meaning that the noise is memoryless and $\mu>1$ indicating that the noise has persistent memory.

\section{RELAY SELECTION TECHNIQUES}

\section{A. Conventional Relay Selection}

For conventional relay selection the best relay out of all the available relays is selected based on the following criterion

$$
R_{b}=\underset{m \in\{1,2, \ldots, M\}}{\arg \max }\left\{\min \left\{\left|h_{S R_{m}}\right|^{2},\left|h_{R_{m} D}\right|^{2}\right\}\right\}
$$


This selection criterion constitutes a tight upper bound for the end-to-end SNR [3]. While this criterion shows the best performance for AWGN environment, this may not be the optimal choice for the considered bursty impulsive noise environment since this criterion is based on the channel quality only and can not utilize the impulsive noise characteristics in the selection process.

\section{B. Genie Selection}

For genie selection it is assumed that the relays know the states of the impulsive noise perfectly. After knowing the state of the impulsive noise at each relay, i.e., after knowing which of the relays were affected by background Gaussian noise and which of the relays were affected by impulsive interferers, a selection strategy would be to eliminate those relays affected by impulsive interferers from the set of available relays in making a best relay selection. The best relay amongst the rest of the relays is then the one with the best end-to-end sourcerelay-destination path which is confirmed by (8). If all the available relays were affected by impulsive noise, the best relay is selected among all the available relays according to (8). It is assumed that the relays can exchange their noise states among each other through gossiping [13]. ${ }^{1}$ Similar to [3], it is also assumed that each relay knows its $S R$ and $R D$ channel gains, and decides which one has the best channel condition among those which are in good state.

While the genie selection approach is important conceptually, it is very hard if not impossible, to know the states of the impulsive noise accurately at the relays. In the following section, we will provide some algorithms which are responsible to obtain the states of the noise that affect the relays.

\section{MAP-based State Detection}

For MAP-based relay selection scheme, to know the states of impulsive noise based on the received sequence, each relay calculates the a posteriori probability $p\left(s_{m, k} \mid y_{S R_{m}}^{K}\right)$ that the state $s_{m, k}$ is the actual channel state of relay $R_{m}$ at time epoch $k$. This can be computed from

$$
p\left(s_{m, k} \mid y_{S R_{m}}^{K}\right) \propto p\left(s_{m, k}, y_{S R_{m}}^{K}\right)
$$

where $y_{S R_{m}}^{K}=\left\{y_{S R_{m}, 0}, y_{S R_{m}, 1}, \ldots, y_{S R_{m}, K-1}\right\}$ is the whole received sequence and $K$ is the size of the sequence. Let us define the following quantities

$$
\begin{gathered}
\alpha_{k}\left(s_{m, k}\right)=p\left(y_{S R_{m}, 0}, y_{S R_{m}, 1}, \ldots, y_{S R_{m}, k-1}, s_{m, k}\right) \\
\beta_{k}\left(s_{m, k}\right)=p\left(y_{S R_{m}, k}, y_{S R_{m}, k+1}, \ldots, y_{S R_{m}, K-1} \mid s_{m, k}\right) \\
\delta_{k}\left(x_{S, k}, s_{m, k}, s_{m, k+1}\right)=p\left(s_{m, k+1} \mid s_{m, k}\right) p\left(n_{S R_{m}, k} \mid s_{m, k}\right)
\end{gathered}
$$

where $\alpha_{k}\left(s_{m, k}\right)$ and $\beta_{k}\left(s_{m, k}\right)$ are referred to as the forward and backward filters and $\delta_{k}\left(x_{S, k}, s_{m, k}, s_{m, k+1}\right)$ represents the branch metrics of the trellis diagram shown in Fig. 2. For a TSMG model, the quantity $p\left(n_{S R_{m}, k} \mid s_{m, k}\right)$ is given by (4) and (5) respectively. Using (10) and (11), the probability term $p\left(s_{m, k}, y_{S R_{m}}^{K}\right)$ in (9) can be written as

$$
p\left(s_{m, k}, y_{S R_{m}}^{K}\right)=\alpha_{k}\left(s_{m, k}\right) \beta_{k}\left(s_{m, k}\right)
$$

\footnotetext{
${ }^{1}$ In this discussion, how the gossip-based algorithm works to gather such information is not important.
}

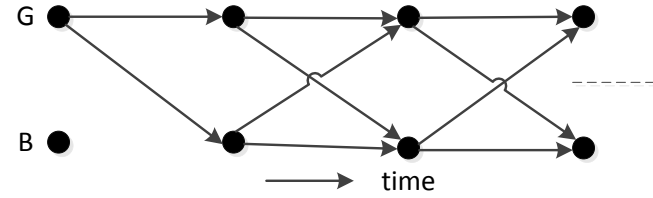

Fig. 2. Trellis representation of the Two-state Markov-Gaussian noise model.

Then the decision rule would be

$$
\hat{s}_{m, k}= \begin{cases}G & \text { if } L_{s_{m, k}} \geq 0 \\ B & \text { if } L_{s_{m, k}}<0\end{cases}
$$

where, $\hat{s}_{m, k}$ is the estimate of the impulsive noise state of relay $m$ at time epoch $k$ and $L_{s_{m, k}}$ is the log-likelihood ratio (LLR). The LLR values at the relays are defined by

$$
L_{s_{m, k}}=\ln \left\{\frac{\alpha_{k}\left(s_{m, k}=G\right) \beta_{k}\left(s_{m, k}=G\right)}{\alpha_{k}\left(s_{m, k}=B\right) \beta_{k}\left(s_{m, k}=B\right)}\right\}
$$

The forward and backward filters can be computed recursively as

$$
\begin{gathered}
\alpha_{k+1}\left(s_{m, k+1}\right)=\sum_{s_{m, k}, x_{S, k}} \alpha_{k}\left(s_{m, k}\right) p\left(x_{S, k}\right) \delta_{k}\left(x_{S, k}, s_{m, k}, s_{m, k+1}\right) \\
\beta_{k}\left(s_{m, k}\right)=\sum_{s_{m, k+1}, x_{S, k}} \beta_{k+1}\left(s_{m, k+1}\right) p\left(x_{S, k}\right) \delta_{k}\left(x_{S, k}, s_{m, k}, s_{m, k+1}\right)
\end{gathered}
$$

where the forward and backward filters are initialized with

$$
\alpha_{0}\left(s_{m, 0}=S\right)=p_{S}, \text { and } \beta_{K}\left(s_{m, K}=S\right)=1 . S \in(G, B)
$$

\section{IID-based State Detection}

For iid-based relay selection scheme, it is assumed that the channels are memoryless [14], that is, when we consider $\mu=1$ in the noise process. For this scheme, the above MAPbased state detection algorithm degenerates in a symbol-bysymbol algorithm, i.e., no recursion should be performed, and the probability of having in a state depends on the probability $p\left(s_{m, k} \mid y_{S R_{m}, k}\right)$ which can be computed from,

$$
\begin{aligned}
& p\left(s_{m, k} \mid y_{S R_{m}, k}\right) \propto p\left(s_{m, k}, y_{S R_{m}, k}\right) \\
= & p\left(s_{m, k}\right) \sum_{x_{S, k}} p\left(n_{S R_{m}, k} \mid s_{m, k}\right) p\left(x_{S, k}\right)
\end{aligned}
$$

For this scheme, the LLR values at the relays are obtained from

$$
L_{s_{m, k}}=\ln \left\{\frac{p\left(s_{m, k}=G\right) \sum_{x_{S, k}} p\left(n_{S R_{m}, k} \mid s_{m, k}=G\right) p\left(x_{S, k}\right)}{p\left(s_{m, k}=B\right) \sum_{x_{S, k}} p\left(n_{S R_{m}, k} \mid s_{m, k}=B\right) p\left(x_{S, k}\right)}\right\}
$$

Based on the LLR values, each relay then decides about the states according to (14).

\section{E. Random Relay Selection}

For random relay selection, one relay out of all the available relays is picked randomly for cooperation. This is the simplest method for single relay selection and probably will show the worse performance since it neither considers the channel quality nor the noise states for relay selection. 


\section{PERFORMANCE ANALYSIS}

In this section, we derive a BER expression of best relay selection in the presence of i.i.d. Rayleigh fading and bursty impulsive noise under certain assumptions. Since it is assumed that a erroneous data can be transmitted from the best relay, the end-to-end probability of error for a given value of $L$ ( $L$ is the cardinality of the set of relays which are in good state) can be expressed as

$$
P_{e}(L)=P_{e, b}(L) \cdot P_{e, S R D}^{e r}(L)+\left(1-P_{e, b}(L)\right) \cdot P_{e, S R D}^{n e r}(L)
$$

where $P_{e, b}(L)$ is the probability of error at the best relay conditioned on $L, P_{e, S R D}^{e r}$ is the probability of error at the destination after combining the two signals coming from the source and the best relay when the error is propagated from the relay, and $P_{e, S R D}^{n e r}$ is the probability of error at the destination when there is no error propagation from the best relay and hence the source and the relay will transmit the same data. For BPSK modulated signal, $P_{e, b}(L)$ is obtained by [15]

$$
\begin{aligned}
P_{e, b}(L)= & \sum_{i=1}^{L}\left(\begin{array}{c}
L \\
i
\end{array}\right) \frac{(-1)^{i-1}}{\bar{\gamma}_{R D}} \frac{i \bar{\gamma}}{i \bar{\gamma}_{S R}-\bar{\gamma}}\left[\varphi\left(\frac{1}{\bar{\gamma}_{S R}}\right)-\varphi\left(\frac{i}{\bar{\gamma}}\right)\right] \\
& +\sum_{i=1}^{L}\left(\begin{array}{c}
L \\
i
\end{array}\right) \frac{i(-1)^{i-1}}{\bar{\gamma}_{S R}} \varphi\left(\frac{i}{\bar{\gamma}}\right)
\end{aligned}
$$

where $\bar{\gamma}_{i j}=\frac{\mathrm{P}_{i} \Omega_{i j}}{\sigma_{C}^{2}}$ incorporates the average received $S N R$ of ij link, $\bar{\gamma}=\frac{\bar{\gamma}_{S R} \bar{\gamma}_{R D}}{\bar{\gamma}_{S R}+\bar{\gamma}_{R D}}$, and

$$
\varphi(\theta)=\frac{1}{2 \theta}\left[1-\frac{1}{\sqrt{1+\theta}}\right]
$$

Hence, the average BER at the best relay is obtained by

$$
P_{e, b}=\sum_{L=1}^{M}\left(\begin{array}{c}
M \\
L
\end{array}\right)\left(1-P_{B}\right)^{L}\left(P_{B}\right)^{M-L} P_{e, b}(L)+P_{B}^{M} P_{e, b}\left(\sigma_{B}^{2}\right)
$$

where $P_{e, b}\left(\sigma_{B}^{2}\right)$ is the probability of error at the best relay when all the relays are in bad state and hence the best relay is selected among them. Since, we don't know what's the error rate performance under that scenario, we upper bound this probability by 0.5 .

In order to determine $P_{e, S R D}^{e r}$ and $P_{e, S R D}^{n e r}$ we have to know which combiner is used for combining the signal coming from the source and the best relay. Since it is assumed that the best relay is selected among the set of the relays which are in good state, we can perform MRC at the destination to combine optimally the signal coming from the $S D$ and $R_{b} D$ paths. For MRC, the SNR after combining the signals is the sum of the SNRs of $S D$ and $R_{b} D$ links and the BER at the destination when there is no error propagated from the relay can be obtained by [15]

$$
\begin{aligned}
P_{e, S R D}^{n e r}= & \sum_{i=1}^{L}\left(\begin{array}{c}
L \\
i
\end{array}\right) \frac{(-1)^{i-1}}{\bar{\gamma}_{S R}} \frac{i \bar{\gamma}}{i \bar{\gamma}_{R D}-\bar{\gamma}}\left\{\frac { \overline { \gamma } _ { R D } } { \overline { \gamma } _ { S D } - \overline { \gamma } _ { R D } } \left[\varphi\left(\frac{1}{\bar{\gamma}_{S D}}\right)\right.\right. \\
& \left.\left.-\varphi\left(\frac{1}{\bar{\gamma}_{R D}}\right)\right]-\frac{\bar{\gamma}}{i \bar{\gamma}_{S D}-\bar{\gamma}}\left[\varphi\left(\frac{1}{\bar{\gamma}_{S D}}\right)-\varphi\left(\frac{i}{\bar{\gamma}}\right)\right]\right\} \\
+ & \sum_{i=1}^{L}\left(\begin{array}{c}
L \\
i
\end{array}\right) \frac{(-1)^{i-1}}{\bar{\gamma}_{R D}} \frac{i \bar{\gamma}}{i \bar{\gamma}_{S D}-\bar{\gamma}}\left[\varphi\left(\frac{1}{\bar{\gamma}_{S D}}\right)-\varphi\left(\frac{i}{\bar{\gamma}}\right)\right](26)
\end{aligned}
$$

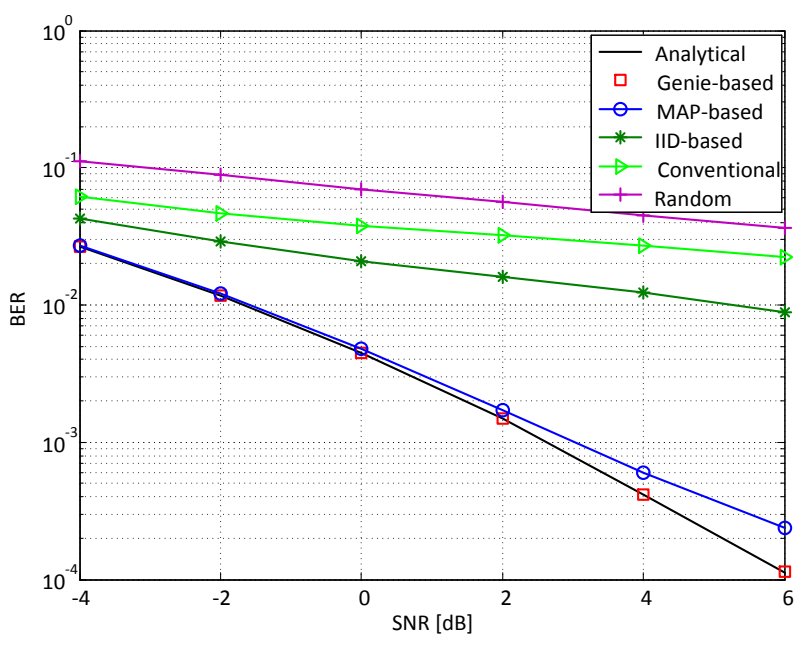

Fig. 3. Analytical and simulated BER performances at the best relay with $M=5$ relays against SNR. A system employing a BPSK modulation is considered and the performance of various relay selection schemes over TSMG channels, each characterized by $p_{B}=0.1, \mu=100, R=100$ is shown.

Also, for BPSK modulated signal $P_{e, S R D}^{e r}$ can be tightly approximated by [15]

$$
P_{e, S R D}^{e r}=\frac{\bar{\gamma}_{R_{b} D}}{\bar{\gamma}_{R_{b} D}+\bar{\gamma}_{S D}}
$$

where $\bar{\gamma}_{R_{b} D}$ is the average received SNR from the best relay to the destination which is equal to [15]

$$
\begin{aligned}
\bar{\gamma}_{R_{b} D}= & \sum_{i=1}^{L}\left(\begin{array}{c}
L \\
i
\end{array}\right) \frac{(-1)^{i-1}}{\bar{\gamma}_{S R}} \frac{i \bar{\gamma}}{i \bar{\gamma}_{R D}-\bar{\gamma}}\left[\bar{\gamma}_{R D}^{2}-\left(\frac{\bar{\gamma}}{i}\right)^{2}\right] \\
& +\sum_{i=1}^{L}\left(\begin{array}{c}
L \\
i
\end{array}\right) \frac{(-1)^{i-1}}{\bar{\gamma}_{R D}} \bar{\gamma}^{2}
\end{aligned}
$$

Therefore, the error probability at the destination for a given value of $L$ is obtained by putting (23), (26), and (27) in (22). Finally, the average BER at the destination is given by

$$
P_{e}=\sum_{L=1}^{M}\left(1-P_{B}\right)^{L}\left(P_{B}\right)^{M-L} P_{e}(L)+P_{B}^{M} P_{e}\left(\sigma_{B}^{2}\right)
$$

where $P_{e}\left(\sigma_{B}^{2}\right)$ is the probability of error at the destination when all the relays are in bad state and hence the best relay is selected among them. Since, we don't know which combiner is optimal under that scenario and what's the error rate performance, we also upper bound this probability by 0.5 .

\section{NUMERICAL RESULTS}

Here, we present the BER performances of DF relay selection schemes where a sequence of equally likely information bits of length 64,800 is mapped onto BPSK modulation sequence and transmitted over TSMG channels characterized by the identical parameters of bad state occurring rate $p_{B}=0.1$, channel memory $\mu=100$, and impulsive to Gaussian noise power ratio $R=100$. In the simulations, it is assumed that both the source and the relay transmit power are equal, and the path loss exponent $\eta=2$. 


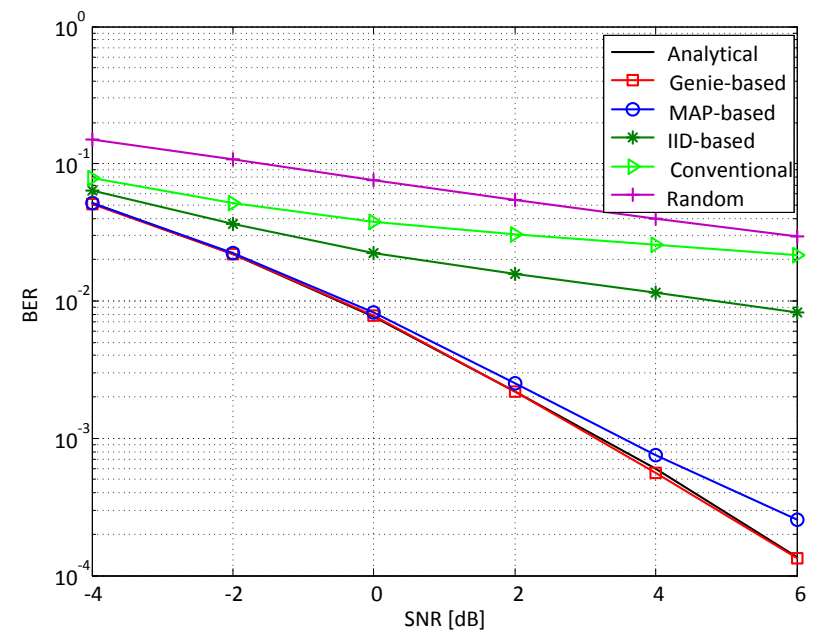

Fig. 4. End-to-end analytical and simulated BER performances of DF relay selection schemes with $M=5$ relays against SNR. A system employing a BPSK modulation is considered and the performance of various relay selection schemes over TSMG channels, each characterized by $p_{B}=0.1, \mu=100$, $R=100$ is shown

Fig. 3 shows the analytical and simulated BER performances at the best relay with $M=5$ relays assuming different relay selection techniques. In the simulations, it is assumed that the distance between the source and the destination is normalized to unity, i.e., $\lambda_{s d}=1$ and the relays are distributed at the distance of $\lambda_{S R_{m}}=0.4, \forall m$. The BER formula in (25) is used to obtain the analytical results at the best relay. For the simulation results, the BER performances are calculated for 2000 frames of 64,800 information bits each against $S N R$. The $S N R$ is defined as, $S N R=\varepsilon\left\{\left|x_{S, k}\right|^{2}\right\} / \sigma_{G}^{2}$, where $\sigma_{G}^{2}$ is the background Gaussian noise power. For the considered BPSK modulated signal, $\varepsilon\left\{\left|x_{s, k}\right|^{2}\right\}$ is equal to one and $\sigma_{G}^{2}$ is adjusted to achieve a given SNR. Also, the SNR is equal to the SNR of the $S D$ link, because the distance between $S$ and $D$ is normalized to unity. From Fig. 3, it is seen that the analytical result perfectly matches with the simulation result obtained in case of genie selection. While the genie selection is hard to implement if not impossible, from the figure it is also seen that the proposed MAP-based relay selection scheme almost approaches the performance of genie selection scheme and performs significantly better than the conventional schemes at the expense of higher complexity due to the MAP detection. This confirms the benefits of utilizing the noise memory in the relay selection process for multi-relay scenario. Also, more simpler iid-based relay selection scheme still achieves better performance that the conventional relay selection scheme by utilizing the impulsive noise statistics in the detection process. Finally, the simplest random relay selection shows the worse performance.

Fig. 4 shows the end-to-end analytical and simulated BER performances of DF cooperative relaying schemes with $M=5$ relays assuming different relay selection techniques. In the simulations, it is assumed that $\lambda_{s d}=1$ and $\lambda_{S R_{m}}=0.4$, $\forall m$. The BER formula in (29) is used to obtain the analytical result for the best relay selection scenario. From Fig. 4, it is seen that similar to the obtained results at the best relay, the same conclusions hold for end-to-end performance also,

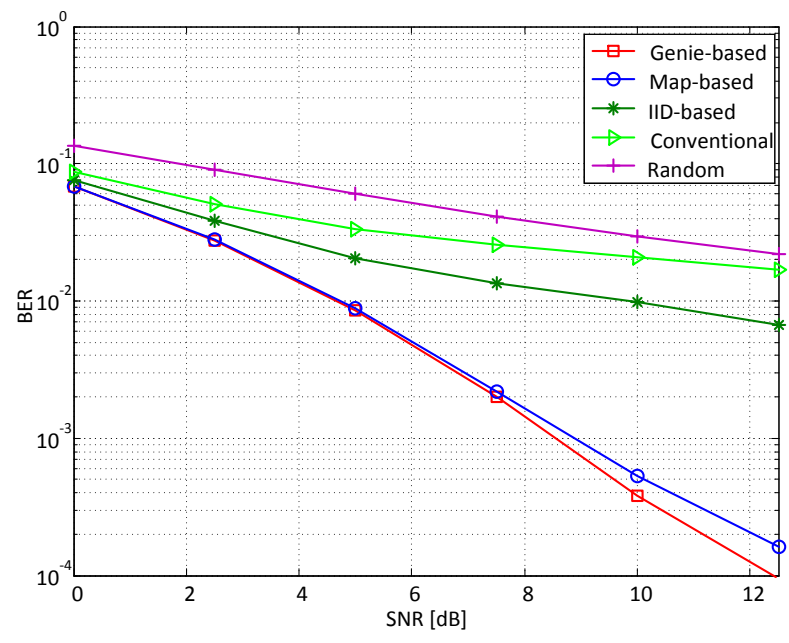

Fig. 5. End-to-end BER performances of DF relay selection schemes for symmetrical relay networks with $M=5$ relays against SNR. A system employing a BPSK modulation is considered and the performance of various relay selection schemes over TSMG channels, each characterized by $p_{B}=0.1$, $\mu=100, R=100$ is shown.

i.e, the end-to-end analytical BER perfectly matches with the simulation result obtained in case of genie selection. From the figure it is further verified that the proposed MAP-based relay selection is an useful tool in decreasing impulsive effects and thus improving the system performance significantly compared to the conventional scheme. Fig. 5 also shows the end-toend BER performances of DF relay selection schemes with $M=5$ relays assuming different relay selection techniques under symmetrical network condition, i.e., when $\lambda_{s d}=1$, $\lambda_{S R_{m}}=1$, and $\lambda_{R_{m} D}=1$. From Fig. 5, we can conclude that the performance improvement of MAP-based relay selection scheme over the conventional schemes is independent of the network geometry.

In order to check the robustness of the proposed relay selection techniques in a different type of noise, we consider $\gamma=1$ in the noise process, which corresponds to the iid case of TSMG noise commonly known as Bernoulli-Gaussian [14] noise in the literature. The BER results for this scheme are provided in Fig. 6. It is observed from the figure that the MAPbased relay selection scheme shows the same performance as the iid-based selection scheme. This is expected since the iidbased scheme is optimal for Bernoulli-Gaussian noise. These results confirm that the MAP-based relay selection scheme reduces to the memoryless scheme when there is no time correlation among the noise samples. Again, the conventional relay selection shows worse performances in all cases.

We also considered systems employing powerful channel codes such as low-density parity check (LDPC) codes. Fig. 7 shows the simulated BER performances of DF relay selection schemes with $M=5$ relays for LDPC coded transmission. It is assumed that a sequence of equally likely information bits of length 32,400 is first encoded using LDPC channel coding based on the DVB-S2 standard with the code rate of $1 / 2$. The coded sequence is then interleaved using a random interleaver and mapped onto BPSK modulation sequence. For LDPC decoding, the number of iterations is set to 50. As 


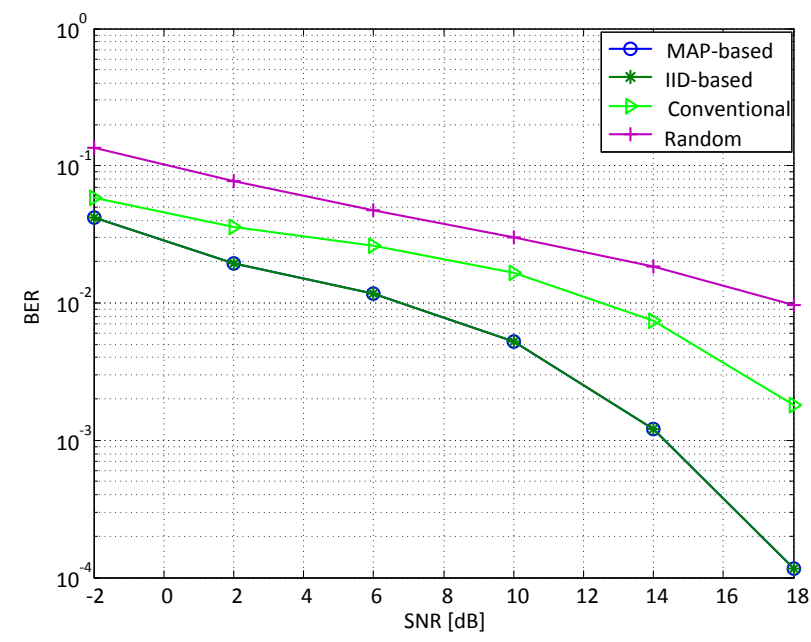

Fig. 6. End-to-end BER performances of DF relay selection schemes over iid Bernoulli-Gaussian noise with $M=5$ relays and $\lambda_{S R_{m}}=0.6, \forall m$ against SNR. A system employing a BPSK modulation is considered and the performance of various relay selection schemes over memoryless channels, each characterized by $p_{B}=0.1, \mu=1, R=100$ is shown.

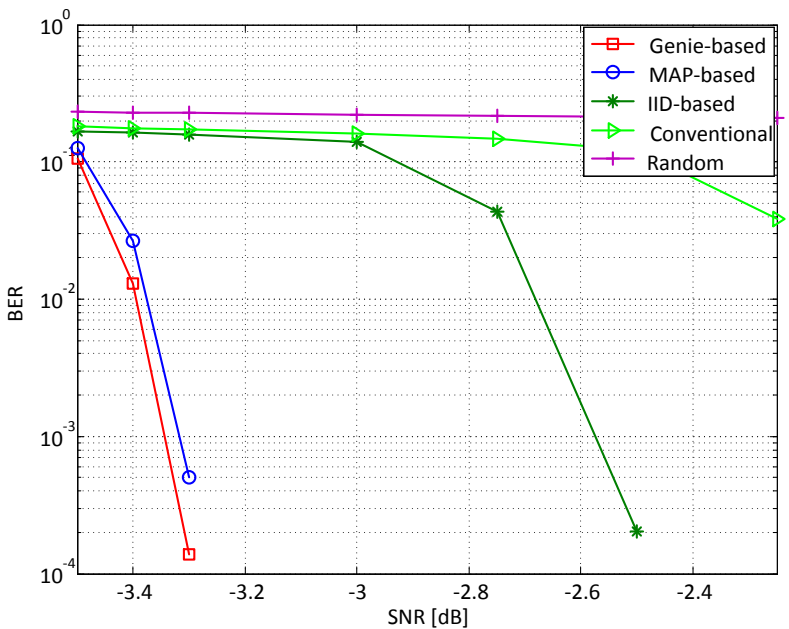

Fig. 7. End-to-end BER performances of DF relay selection schemes with $M=5$ relays against SNR under coded transmission. A system employing a BPSK modulation is considered and the performance of various relay selection schemes over TSMG channels, each characterized by $p_{B}=0.1, \mu=100$, $R=100$ is shown.

expected, from Fig. 7, it is observed that similar to uncoded transmission, significant performance gain is achieved when the noise memory is taken into account in the relay selection process.

\section{CONCLUSION}

In this paper, we have investigated the performance of some conventional relay selection techniques in impulsive noise environment in the presence of Rayleigh fading and propose an improved approach for relay selection which is based on both the channel state information of source-relay and relaydestination links and the state of the impulsive noise that affect those links. We also have investigated the BER formula for the proposed scenario. From the obtained results it is seen that, the analytical results agree with the simulations. Also, our proposed MAP-based relay selection scheme performs significantly better than the conventional schemes developed for AWGN channel, and that neglects the noise memory.

\section{ACKNOWLEDGMENT}

This work was supported by Hydro-Quebec, the Natural Sciences and Engineering Research Council of Canada, and McGill University in the framework of the NSERC/HydroQuebec/McGill Industrial Research Chair in Interactive Information Infrastructure for the Power Grid.

\section{REFERENCES}

[1] A. Nosratinia, T. E. Hunter, and A. Hedayat, "Cooperative communication in wireless networks," IEEE Communications Magazine, vol. 42, no. 10, pp. 74-80, 2004.

[2] J. N. Laneman, D. N. Tse, and G. W. Wornell, "Cooperative diversity in wireless networks: efficient protocols and outage behavior," IEEE Transactions on Information Theory, vol. 50, no. 12, pp. 3062-3080, 2004.

[3] A. Bletsas, A. Khisti, D. P. Reed, and A. Lippman, "A simple cooperative diversity method based on network path selection," IEEE Journal on Selected Areas in Communications, vol. 24, no. 3, pp. 659-672, 2006.

[4] A. S. Ibrahim, A. K. Sadek, W. Su, and K. R. Liu, "Cooperative communications with relay-selection: when to cooperate and whom to cooperate with?" IEEE Transactions on Wireless Communications, vol. 7, no. 7, pp. 2814-2827, 2008.

[5] M. M. Fareed and M. Uysal, "On relay selection for decode-and-forward relaying," IEEE Transactions on Wireless Communications, vol. 8, no. 7, pp. 3341-3346, 2009.

[6] D. Middleton, "Statistical-physical models of electromagnetic interference," IEEE Transactions on Electromagnetic Compatibility, vol. EMC19, no. 3, pp. 106-127, 1977.

[7] F. Sacuto, B. L. Agba, F. Gagnon, and F. Labeau, "Evolution of the RF characteristics of the impulsive noise in high voltage environment," in IEEE Third International Conference on Smart Grid Communications. IEEE, 2012, pp. 686-691.

[8] M. Zimmermann and K. Dostert, "Analysis and modeling of impulsive noise in broad-band powerline communications," IEEE Transactions on Electromagnetic Compatibility, vol. 44, no. 1, pp. 249-258, 2002.

[9] I. Krikidis, J. S. Thompson, S. McLaughlin, and N. Goertz, "Max-min relay selection for legacy amplify-and-forward systems with interference," IEEE Transactions on Wireless Communications, vol. 8, no. 6, pp. 3016-3027, 2009.

[10] A. Nasri and R. Schober, "Performance of cooperative diversity systems in non-Gaussian environments," in IEEE International Conference on Communications. IEEE, 2010, pp. 1-6.

[11] L. Bahl, J. Cocke, F. Jelinek, and J. Raviv, "Optimal decoding of linear codes for minimizing symbol error rate (corresp.)," IEEE Transactions on Information Theory, vol. 20, no. 2, pp. 284-287, 1974.

[12] D. Fertonani and G. Colavolpe, "On reliable communications over channels impaired by bursty impulse noise," IEEE Transactions on Communications, vol. 57, no. 7, pp. 2024-2030, 2009.

[13] Z. J. Haas, J. Y. Halpern, and L. Li, "Gossip-based ad hoc routing," IEEE/ACM Transactions on Networking (ToN), vol. 14, no. 3, pp. 479491, 2006.

[14] M. Ghosh, "Analysis of the effect of impulse noise on multicarrier and single carrier QAM systems," IEEE Transactions on Communications, vol. 44, no. 2, pp. 145-147, 1996.

[15] K. Tourki, H.-C. Yang, M.-S. Alouini, and K. A. Qaraqe, "New results on performance analysis of opportunistic regenerative relaying," Physical Communication, vol. 9, pp. 97-111, 2013. 